occurred at 137 centers throughout the world in the phase 3 trial, which suggests that the skills necessary to safely administer the combination therapy are widely available.

Furthermore, and to Valsecchi's point, among the 120 patients who discontinued combination therapy because of toxic effects, the response rate was $67.5 \%$. To us, this supports the current guidelines for management of toxic effects and discontinuation of treatment, since it shows that high response rates can be observed in the context of no treatment-related deaths. Longer followup will be needed to assess the effect of treatment discontinuation on overall survival.
James Larkin, M.D., Ph.D.

Royal Marsden Hospital

London, United Kingdom

F. Stephen Hodi, M.D.

Dana-Farber Cancer Institute

Boston, MA

Jedd D. Wolchok, M.D., Ph.D.

Memorial Sloan Kettering Cancer Center

New York, NY

wolchokj@mskcc.org

Since publication of their article, the authors report no further potential conflict of interest.

1. Postow MA, Chesney J, Pavlick AC, et al. Nivolumab and ipilimumab versus ipilimumab in untreated melanoma. $\mathrm{N}$ Engl J Med 2015;372:2006-17.

DOI: $10.1056 / N E J M c 1509660$

\title{
Long-Term Use of Ticagrelor in Patients with Prior Myocardial Infarction
}

TO THE EDITOR: In their report on the PEGASUSTIMI 54 (Prevention of Cardiovascular Events in Patients with Prior Heart Attack using Ticagrelor Compared to Placebo on the Background of Aspirin-Thrombolysis in Myocardial Infarction 54) trial, Bonaca et al. (May 7 issue) ${ }^{1}$ suggest that the benefit of dual antiplatelet therapy comes at a cost of an increased risk of bleeding events. A dual antiplatelet regimen administered at an even lower dose than the one used in this study could confer a reduced risk of bleeding events and improved secondary prophylaxis against recurrent ischemic events as compared with aspirin.

We successfully tested a strategy of alternateday administration of clopidogrel 1 year after percutaneous coronary intervention with placement of a drug-eluting stent. ${ }^{2}$ We hypothesized that the degree of antiplatelet effect that was required to prevent very late stent thrombosis decreases with time as the stent undergoes endothelialization - in other words, the therapeutic threshold that is required to prevent very late stent thrombosis decreases with time. The antiplatelet effect of clopidogrel lasts 5 to 7 days. Typically, after the interruption of clopidogrel therapy, stent thrombosis occurs after 3 to 4 days, which signifies the recovery of enough platelet function to produce stent thrombosis (i.e., above the therapeutic threshold). The use of clopidogrel every other day or a lower dose of ticagrelor among patients receiving a drug-eluting stent may be sufficient to cross the much reduced therapeutic threshold that is required to prevent very late stent thrombosis after 1 year and to avert recurrent coronary events from spontaneous plaque rupture without much bleeding risk (a so-called Goldilocks dose).

Sanjiv Sharma, M.D.

Bakersfield Heart Hospital

Bakersfield, CA

sanjiv1122@yahoo.com

Navneet Kaur, M.D.

Central Cardiology Medical Clinic

Bakersfield, CA

\section{Rohan Sharma}

University of California, Los Angeles

Los Angeles, CA

Dr. Sharma reports receiving lecture fees from Eli Lilly and Daiichi Sankyo and being an investigator for the PEGASUS-TIMI 54 trial. No other potential conflict of interest relevant to this letter was reported.

1. Bonaca MP, Bhatt DL, Cohen M, et al. Long-term use of ticagrelor in patients with prior myocardial infarction. $\mathrm{N}$ Engl J Med 2015;372:1791-800.

2. Sharma S, Forrester JS. A novel regimen of alternate day clopidogrel would provide a cost-effective strategy to prevent very late stent thrombosis. Med Hypotheses 2012;78:166-70.

DOI: $10.1056 / N E J M c 1508692$

TO THE EDITOR: Bonaca et al. report higher rates of cancer-related death among patients receiving either $90 \mathrm{mg}$ or $60 \mathrm{mg}$ of ticagrelor than among those receiving placebo $(1.10 \%$ and $0.92 \%$ vs. $0.76 \%$ ) (Table S2 in the Supplementary Appendix, 


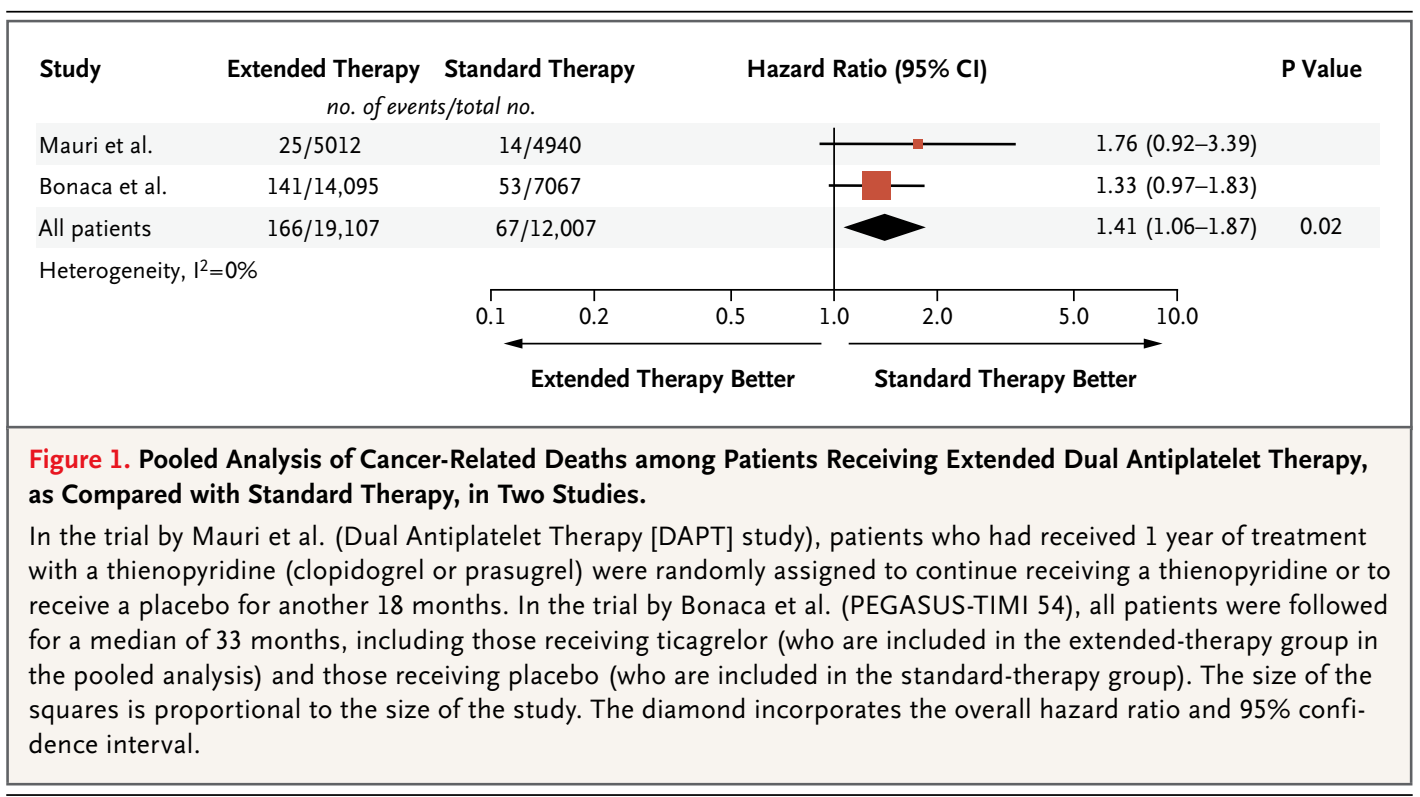

available with the full text of the article at NEJM.org). Similar findings have been reported by Mauri et al. ${ }^{1}$ with an extended use of thienopyridines beyond 1 year.

A pooled analysis of these two studies suggests a significant relative increase of $41 \%$ in the number of cancer-related deaths among patients who were treated with extended dual antiplatelet therapy (Fig. 1). In this analysis, the number of patients who would need to be treated to cause one cancer death was 322. Could the author indicate whether there was an imbalance in the diagnosis of cancer before and after randomization in the two study groups? If this is not the case, the reason for an increase in cancer-related deaths with an extended duration of dual antiplatelet therapy should be investigated.

Francesco Costa, M.D.

Erasmus Medical Center

Rotterdam, the Netherlands

Marco Valgimigli, M.D., Ph.D.

Swiss Cardiovascular Center

Bern, Switzerland

marco.valgimigli@insel.ch

No potential conflict of interest relevant to this letter was reported.

1. Mauri L, Kereiakes DJ, Yeh RW, et al. Twelve or 30 months of dual antiplatelet therapy after drug-eluting stents. $\mathrm{N}$ Engl J Med 2014:371:2155-66.

DOI: $10.1056 / N E J M c 1508692$
TO THE EDITOR: Bonaca et al. are missing the most important finding of the PEGASUS-TIMI 54 study - namely, that drugs that cause excess bleeding also increase the risk of solid cancers. This study showed an increasing dose-response relationship for deaths from cancer: 77 in the group receiving $90 \mathrm{mg}$ of ticagrelor and 64 in those receiving $60 \mathrm{mg}$ of ticagrelor, as compared with 53 receiving placebo. In the Study of Platelet Inhibition and Patient Outcomes (PLATO), the administration of ticagrelor was too short and the follow-up was too incomplete to provide reliable data about cancer. ${ }^{1}$

A review of prasugrel by the Food and Drug Administration (FDA) ${ }^{2}$ first reported the association between bleeding and solid cancers. In the Trial to Assess Improvement in Therapeutic Outcomes by Optimizing Platelet Inhibition with Prasugrel-Thrombolysis in Myocardial Infarction 38 (TRITON-TIMI 38), ${ }^{3}$ the hazard ratios in the prasugrel group, as compared with the clopidogrel group, for the incidence of solid cancers and death were both approximately 1.6 $(\mathrm{P}=0.001)$. An FDA review ${ }^{4}$ of the DAPT study documented that the relative risk of the incidence of a solid cancer was about 1.2 with clopidogrel and 1.3 with prasugrel, as compared with placebo. The rates of cancer-related death (relative risk, 2.2; $\mathrm{P}=0.02$ ) were higher with continued use of thienopyridines. ${ }^{5}$ The latter FDA review ${ }^{4}$ also provides an 